\section{Measurement Theory}

Michael Franzen

Allegheny General Hospital, Pittsburgh, PA, USA

\section{Definition}

Measurement theory is a complex topic, but in psychological research it usually refers to the type of scale used to obtain numbers to represent values of the variable of interest. These types of scales are nominal categorical, ordinal, interval, or ratio. In the nominal scale, values assigned to the variable refer to characteristics of the variable for which greater or lesser numbers do not refer to qualities of the observation. For example, in measuring gender, males might be assigned the number "one," and females might be assigned the number "two." These numbers refer to values of the variable but do not refer to any inherent quantitative qualities of the observations. In ordinal measurement, the relative values of the numbers refer to characteristics of the observations. A subject with a value of "two" for some variables has more of that variable than a subject with a values of "one." However, the difference between a value of 6 and a value of 8 is not the same as the difference between a value of 4 and a value of 6 . When that requirement can be filled, the scale has interval qualities. This refers to the fact that equalsized intervals represent equal-sized differences in the observations. Finally, when different values of the observation represent corresponding different amounts of the variable, the scale is said to be ratio. Here a value of 4 is twice the value of 2 , and a value of 300 is twice the value of 150 .

\section{References and Readings}

Granberg-Rademacker, J. S. (2010). An algorithm for converting ordinal scale measurement data to interval/ ratio scale. Educational and Psychological Measurement, 70, 74-90.

Michell, J. (1986). Measurement scales and statistics: A clash of paradigms. Psychological Bulletin, 100, 398-407. 